International Journal of Engineering \&Technology, $7(1.7)(2018)$ 65-67
International Journal of Engineering \& Technology
WPC
Website: www.sciencepubco.com/index.php/IJET
Review paper

\title{
Supportive caching strategies for MANET: a review
}

\author{
N. Phani Kumar ${ }^{1 *}$, C. Amuthadevi ${ }^{2}$ \\ ${ }^{1}$ Research scholar, Department of Computer Science and Engineering, School of Computing, Vel Tech Rangarajan \\ Dr. Sagunthala R\&D Institute of Science and Technology, Avadi, Chennai-62, TamilNadu, India \\ ${ }^{2}$ Associate Professor, Department of Computer Science and Engineering, School of Computing, Vel Tech Rangarajan \\ Dr. Sagunthala R\&D Institute of Science and Technology, Avadi, Chennai-62, TamilNadu, India \\ *Corresponding author E-mail: phanikumar.nagaram@gmail.com
}

\begin{abstract}
Caching in mobile computing surroundings is a capable approach which could improve information get right of entry to performance and lessen the heavy verbal exchange between client and server. Caching permits sharing and coordination of reserved records between cell hosts. In this paper all the caching schemes for wired, ad hoc and internet mobile advert hoc community are mentioned and compared in terms of cache resolution and cache substitute coverage.
\end{abstract}

Keywords: Caching; Mobile Networks; Mobility; Wireless; Bandwidth.

\section{Introduction}

A mobile ad-hoc system (Manet) is a self-designing system of cell hubs. Every one of the hubs are switches associated through Wi-Fi joins. The switches are detached to circle and arrange themselves subjectively, thusly; the group topology may likewise interchange quickly and erratically. Such a group may furthermore perform in an independent manner, or may moreover be Related to the bigger web or might be utilized as a cross breed Wi-Fi arrange. Mantes have attracted tons intrigue most recent years due to the bendy organizing arrangement it presents [1]. In such systems, cell hubs, normally battery-controlled, Speak with each extraordinary through remote medium and multi-bounce courses. How-Ever, data get admission to applications in Mantes need to confront bring down insights accessibility and higher inspire admission to cost expedited by utilizing the one of a kind capacities of Ma-nets: remote medium, Multi-bounce steering, dynamic topologies, and asset limitations. In Manets getting to offerings and actualities over the group can be moderate and accordingly, storing much of the time got to records is an intense approach for improving in advance with performance. On the off chance that storing data on one gadget is utilized by utilizing itself, this component does now not star vide a consistent system for permitting every other contraption in the system to bene-sound from this data. Consequently, formulating an intense reserving technique for the entire Manet is of extraordinary criticalness since it lets in for improving the execution of the Network as a whole.

\section{Caching}

Reserving is to copy a piece of the information from the data organization to a littler and snappier carport device (store) mediated between the records purchaser and the realities backer, all together that future data gets to might be settled from the reserve with less cost[2].

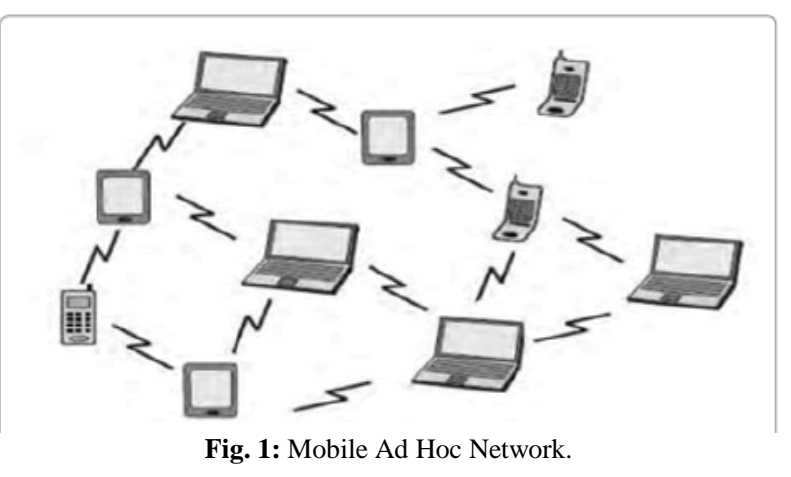

\subsection{Significance of caching}

With reserving, the information motivate admission to put off is diminished since data get to solicitations can be served from the area store, accordingly forestalling the need for records transmission over the rare remote connections. Be that as it may storing systems used in one-jump cellular condition won't not be relevant to multi-bounce specially appointed condition[5]. Since the data or demand may likewise need to travel through two or three jumps. Variable records length, visit measurements refreshes, obliged buyer sources, lacking remote data transmission and customers' versatility make store administration a troublesome errand in cell impromptu net-works.

\subsection{Supportive caching}

As cell hubs in advert hoc systems can likewise have practically identical obligations and extent regular side interest, agreeable reserving, which lets in the sharing and coordination of stored records among two or three hubs, can be utilized to decrease the transfer speed and power consumption. Agreeable storing procedures were generally contemplated in wired systems while little has been proficient to apply this strategy to advert hoc net-works [7]. Because of portability and help imperatives, systems intended 
for focused on net-works may not be applicable to advert hoc net divider right. For instance, most research on co-agent reserving in the web surroundings accept a set topology, yet this couldn't be the situation in advert hoc systems be-reason for portability [9]. For the reason that estimation of the Wi-Fi hyperlink is restrictive from the focused on hyperlink, the selection with respect to where to reserve the data and the best approach to get the stored realities might be unprecedented. By utilizing agreeably storing every now and again got to measurements, cell gadgets do now not always should send solicitations to the realities source.

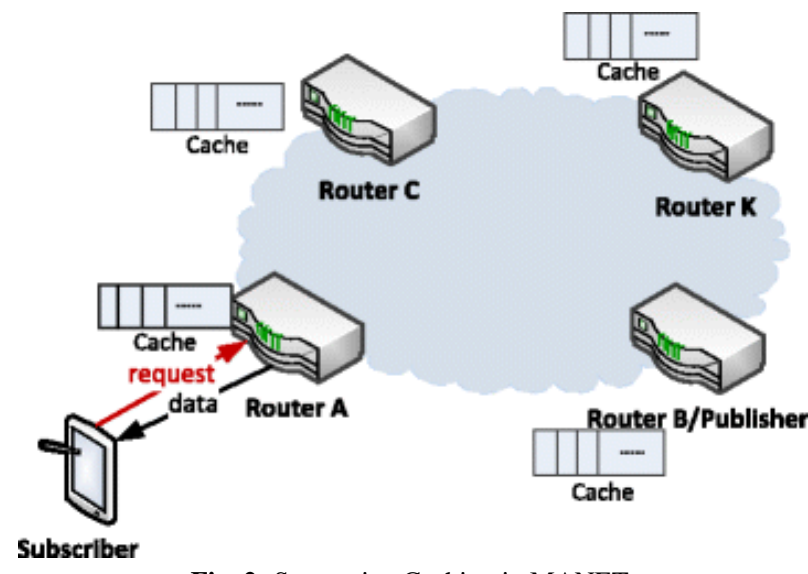

Fig. 2: Supportive Caching in MANET.

The Supportive caching method in proposes 3 strategies of caching. They are cache records, cache course and hybrid cache. In cache records, the intermediate nodes cache the records to serve future requests in preference to fetching records from the statistics center. In cache route, the nodes cache the data route and use it to redirect future requests. As the nodes are cell cache path might not be dependable. For that reason the records route is cached most effective if the caching node may be very close or it outcomes in stale path. In hybrid caching, a node caches the statistics or route primarily based on the information length and time-to-stay (ttl). If data length or ttl is small, cache statistics is most efficient as facts item occupies much less area or the information will become invalid soon. If $\mathrm{ttl}$ or records is huge, cache path is used. But the cache course may be invalid as the nodes are mobile. as a result the hybrid cache makes use of the blessings of both cache statistics and cache course.

\subsection{Push and pull method}

The two essential sorts of reserve sharing methodologies are push based and pull based. With push-basically based reserve sharing, while a hub procures and stores another information question, it effectively publicizes the storing occasion to the hubs in its group. Versatile hubs inside the region will record the storing records after accepting such a promotion vertisement and utilize it to coordinate next solicitations for the equivalent question. This plan supplements the handiness of the stored substance [11]. The value we should pay is the communication overhead for the promotion; a notice is futile if no requirements for the reserved question get up inside the area. In the push-based absolutely conspire, the caching measurements perceived to a hub may moreover wind up obsolete because of hub portability or reserve elective. The draw based technique may moreover vanquish this problem. With pull based reserve sharing, while a phone hub wants to get admission to a records thing that isn't generally stored locally it'll communicate a demand to the hubs in its region. A close-by hub that has stored the information will send a duplicate of the data to the demand originator (a draw task). As opposed to pushing, pulling grants the hub to use the fresh out of the box new store substance. be that as it may, conversely with the pushing procedure, the pulling plan has two disadvantages:

1) On the off chance that the asked for insights thing isn't stored by using any hub inside the territory, the requester hub will sit tight for the time-out $\mathrm{c}$ dialect to terminate sooner than it continues to send some other demand to the data center. This will rationale more prominent get right of section to inertness, and the pulling exertion is in futile.

2) Pulling resorts to communicate to find a stored reproduction of a thing. What's more, numerous copies can have returned to the demand originator if more than one hubs in the community reserve the wished actualities. This presents more prominent correspondence overhead. Some other trouble of problem is the confined reserve space that will be had in a cellular hub. In this manner, a reserve substitution system should be in zone for expelling records objects from the store to prepare for a recently got one, while the reserve is full. Considering the way that store substance of a hub are shared by methods for various hubs, a great reserve substitute scope need to think about the entrance needs from the total group.

\section{COOP -A supportive caching technique in MANETs}

A singular cooperative caching scheme for on-call for records get admission to packages in Manets. The goal is to improve records availability and get admission to performance by using participating local assets of cell nodes. The cooperation of caching nodes is two fold. First, a caching node can solution the facts requests from other nodes. 2nd, a caching node shops the data no longer simplest on behalf of its own desires, however additionally based on different nodes' wishes. COOP addresses fundamental troubles for cooperative caching in Manets:

Cache resolution - how does a cell tool determine where to fetch a information object requested via the consumer?

Cache management - how does a mobile tool determine which facts object to region/purge in its nearby cache? for cache decision, coop attempts to discover a information supply which induces much less communication cost by using making use of ancient profiles and forwarding nodes. For cache control, coop minimizes caching duplications among neighbor nodes and allows cooperative caches to store greater one of a kind facts items to enhance the general overall performance.

\subsection{Limitation of COOP}

A particular helpful storing plans for available to come back to work for records motivate admission to bundles in Manets. The objective is to enhance records accessibility and get admission to performance by utilizing taking an interest nearby resources of cell hubs. The collaboration of reserving hubs is two creases. Initial, a storing hub would solution be able to the realities demands from different hubs. Second, a reserving hub shops the information no longer least difficult in the interest of its own wants, however furthermore in view of various hubs' desires. COOP tends to crucial inconveniences for agreeable storing in Manets:

1) Store determination

How does a cell apparatus figure out where to get a data protest asked for through the shopper?

2) Reserve administration

How does a versatile device figure out which realities protest area/cleanse in its adjacent store? for store choice, coop endeavors to find a data supply which prompts significantly less correspondence cost by utilizing making utilization of old profiles and sending hubs. For reserve control, coop limits storing duplications among neighbor hubs and enables helpful reserves to store more prominent unique actualities things to improve the general execution. 


\section{Conclusion}

On this paper we have mentioned cache sharing troubles related to mobile adhoc network environment and provide analysis of some popular cooperative caching schemes. These caching schemes are useful in Manet environment. Right here we present how these schemes are fantastic so that it will discover a statistics object in a manet by way of the usage of much less sources (e.g. network bandwidth, electricity etc.) and improves the overall performance(statistics availability and latency time). We also mentioned the constraints of these strategies. As the cooperative caching is a useful method to enhance the records availability inside the manet so these analyses might be helpful for the destiny studies.

\section{References}

[1] Augustine A, Sebastian EJ (2016) Routing Mechanism for Mobile Ad Hoc Networks with Improved Security Features. J TelecommunSyst Manage 5:130. https://doi.org/10.4172/21670919.1000130.

[2] Yupapin PP (2014) Human Ad Hoc Networks using Telepathic $\begin{array}{llll}\text { Connections. } & \mathrm{J} & \text { BiosensBioelectron } & \end{array}$ https://doi.org/10.4172/2155-6210.1000e129.

[3] https://www.omicsonline.org/open-access/a-novel-hybrid-routingprotocol-for-mobile-adhoc-network-0976-4860-1-185196.pdf.php?aid=35440.

[4] Batth KK, Singh R (2017) Performance Evaluation of Ant Colony Optimization Based Routing Algorithms for Mobile Ad Hoc Networks. Int J AdvTechnol 8:181. https://doi.org/10.4172/0976$\underline{4860.1000181}$

[5] Aminian M, Naji HR (2013) A Hospital Healthcare Monitoring System Using Wireless Sensor Networks. J Health Med Inform 4:121. https://doi.org/10.4172/2157-7420.1000121.

[6] Sunil Taneja, Ashwani KushAndSima Singh (2016) Encryption Scheme for Secure Routing in Ad Hoc Networks. International Journal of Advancements in Technology.

[7] Thakare P.P., Joshi M.A. And Raut A.D (2012) A Review Paper On Routing Protocols Of Wireless Ad-Hoc Network Technology. International Journal of Networking Issn: 2249-278x \& E-Issn: 2249-2798, Volume 2, Issue 1, 2012, Pp.-35-39.

[8] Ibukunola. A.Modupe, Oludayo. O.OlugbarabAbiodun.Modupe (2013) Minimizing Energy Consumption in Wireless Ad hoc Networks with Meta heuristics. Procedia Computer Science

[9] Volume 19, 2013, Pages 106-115.

[10] Stagewise Arithmetic Orthogonal Matching Pursuit. International Journal of Wireless Information Networks.

[11] ShihongDuan, Tianqing Yu, Jie He (2018) WiDriver: Driver Activity Recognition System Based on WiFi CSI. International Journal of Wireless Information Networks.

[12] https://pdfs.semanticscholar.org/2cad/dbbf77d651897ec4438373e4 b74caf2399c9.pdf 